\title{
Plastome comparison and phylogenomic of Fagopyrum (Polygonaceae): insights into detailed sequence divergence and taxonomic relationships
}

Qiu-jie Li

Sichuan University

Yu Liu

Sichuan University

An-hu Wang

Xichang University

Qing-fu Chen

Guizhou Normal University

Jian-mei Wang

Sichuan University

Lu Peng

Sichuan University

Yi Yang ( $\nabla$ yangyi528@scu.edu.cn )

Sichuan University

\section{Research Article}

Keywords: Fagopyrum, Plastome variation, Phylogenomics, Polygonaceae, Inverted repeat boundary, Genome size, Species relationship

Posted Date: March 1st, 2022

DOI: https://doi.org/10.21203/rs.3.rs-1290200/v1

License: (c) (1) This work is licensed under a Creative Commons Attribution 4.0 International License. Read Full License 


\section{Abstract}

Background: Fagopyrum (Polygonaceae) is a small plant lineage comprised of more than fifteen economically and medicinally important species. However, the phylogenetic relationships of the genus are not well explored, and the characteristics of Fagopyrum chloroplast genomes (plastomes) remain poorly understood so far. It restricts the comprehension of species diversity in Fagopyrum. Therefore, a comparative plastome analysis and comprehensive phylogenomic analyses are required to reveal the taxonomic relationship among species of Fagopyrum.

Results: In the current study, 12 plastomes were sequenced and assembled from eight species and two varieties of Fagopyrum. In the comparative analysis and phylogenetic analysis, eight previously published plastomes of Fagopyrum were also included. A total of 49 plastomes of other genera in Polygonaceae were retrieved from GenBank and used for comparative analysis with Fagopyrum. The variation of the Fagopyrum plastomes is mainly reflected in the size and boundaries of inverted repeat/single copy (IR/SC) regions. Fagopyrum is a relatively basal taxon in the phylogenomic framework of Polygonaceae comprising a relatively smaller plastome size $(158,768-159,985 \mathrm{bp})$ than another genus of Polygonaceae (158,851-170,232 bp). A few genera of Polygonaceae have nested distribution of the IR/SC boundary variations. Although most species of Fagopyrum show the same IRb/SC boundary with species of Polygonaceae, only a few species show different IRa/SC boundaries. The phylogenomic analyses of Fagopyrum supported the cymosum and urophyllum groups and resolved the systematic position of subclades within the urophyllum group. Moreover, the repeat sequence types and numbers were found different between groups of Fagopyrum. The plastome sequence similarity showed significant differences between intra-group and inter-group.

Conclusions: The deletions of intergenic regions cause a short length of Fagopyrum plastomes, which may be the main reason for plastome size diversity in Polygonaceae species. Most of the Polygonaceae plastomes may have experienced gene loss events, resulting in the nested distribution of IR/SC boundary variation. The phylogenomic reconstruction combined with the characteristics comparison of plastomes supports grouping within Fagopyrum. The outcome of these genome resources may facilitate the taxonomy, germplasm resources identification as well as plant breeding of Fagopyrum.

\section{Background}

The genus Fagopyrum belongs to the family Polygonaceae and includes approximately 15 to 28 species [1-5]. Most of the wild species of Fagopyrum are narrowly distributed in mountainous areas of southwest China. Several endemic species are only distributed to the southeastern edge of the QinghaiTibetan Plateau $[2,4]$, and this area is considered as the birthplace of the two cultivated buckwheat species, namely Fagopyrum esculentum and F. tataricum [6]. The known cultivated species F. esculentum, is a cereal that produce gluten-free grains [7]. Additionally, the seeds of Tartary buckwheat (F. tataricum) are used as important functional food ingredients [8], and the rhizome of $F$. cymosum ( $F$. dibotrys) is used as a pharmaceutical drug in lung diseases treatment [9]. The genus Fagopyrum has morphological 
diversity with annual and perennial plants, including herbs, woody lianas, and shrubs (Fig. 1). Plants in the genus Fagopyrum have attracted the attention of botanists because of the economic importance of their cultivars and the potential use of their wild genetic resources [10-11].

The assessment of the phylogenetic relationships among Fagopyrum species is a prerequisite of an efficient breeding program, as it provides a basis for selecting desirable genotypes [12]. In the classical taxonomy based on the inflorescence, coverage of the achene with the perianth, and achene size, the genus Fagopyrum is divided into two major taxa, i.e., F. esculentum sensu lato and F. gilesii sensu lato [13-14]. In phylogenetic studies based on DNA sequences of ITS, $r b c L$, and $a c c D$, Fagopyrum is classified into two groups, i.e., cymosum group and urophyllum group, which are in agreement with the two taxa described in earlier taxonomic studies [12,15-16]. The cymosum group corresponds to $F$. esculentum sensu lato and includes the species F. cymosum, F. esculentum, and F. tataricum. The urophyllum group corresponds to F. gilesii sensu lato and includes the species F. gilesii, F. gracilipes, F. leptopodum, F. statice, and F. urophyllum. Recent studies have also supported this division in Fagopyrum [2, 4, 17]; for example, the species belonging to the big-achene group are distinguished from those in the small-achene group in terms of their persistent perianths, which are longer than the seeds, as well as their larger seeds [2]. Additionally, based on the DNA sequences of $p s b E-p s b L$ and $n d h A$ intron the Fagopyrum species were divided into the cultivated group and the wild group by the phylogenetics [17]. Although these findings demonstrate the existence of two distinct groups in Fagopyrum, due to the limitation associated with plant materials or robust molecular data, the relationships among the species remain obscure within these two groups [4].

In recent years, new species of Fagopyrum have been reported based on morphological characteristics [5, 18-23]. F. hailuogouense, reported as a new species of Fagopyrum, was later shown to belong to the genus Bistorta using molecular evidence [24-25]. F. wenchuanense was originally reported to be closely related to $F$. gracilipes and classified into the urophyllum group, but it was later categorized into the cymosum group based on molecular data $[12,22]$. Therefore, molecular data are required to support the taxonomic status of these new species.

Chloroplast genomes (plastomes) are rich sources of phylogenetic data and are widely used in phylogenetic studies due to their moderate nucleotide substitution rates, uniparental inheritance, conserved structure, ease of sequencing, as well as great abundance of existing data [26-28]. The molecular markers such as highly divergent regions and repeat sequences derived from the entire plastome sequence hold promise for use in species delimitation and population genetics [29-31]. Moreover, the comparison of complete plastome sequences further provides the opportunity to explore sequence variation and molecular evolutionary patterns associated with gene loss, rearrangements, duplication, and transfer events [32-34]. Until now, plastomes have only been used for comparative analyses of single species of Fagopyrum or a few species of the genus. Compared with phylogenetic studies limited to a few complete plastomes or a few plastid loci, plastome phylogenomic studies provide much greater resolution [38-40]. Thus, a comprehensive plastome analysis in Fagopyrum is much needed at the infrageneric level. 
In contrast to previous studies, we sampled a taxonomically representative set of species within the cymosum and the urophyllum group in Fagopyrum. To study the characteristics, comparative plastome analyses were used 20 plastomes of Fagopyrum and 49 other genus plastomes of Polygonaceae (Table S1, S2). The phylogenomic analyses were performed to explore systematic positions and relationships of species in Fagopyrum. The objectives of this study included (1) characterization of the plastome variation of Fagopyrum; (2) to test whether the plastome data could resolve current uncertainties in the phylogeny of the Fagopyrum; (3) to investigate the genetic diversity of Fagopyrum useful in the identification of wild germplasm resources and improvement of cultivated variety and breed.

\section{Results}

\section{Characteristics of plastomes}

A total of 12 Fagopyrum plastomes were obtained in our study. The number of paired-end raw reads obtained by the Illumina sequencing ranged from $6,778,507$ to $25,542,740$. The number of reads mapped to Fagopyrum plastomes ranged from 446, 898 to 2, 356, 508 and the Average coverage depth ranges from $426 \times$ to $5265 \times$ (Table 1 ). The 12 high-quality plastome sequences were deposited in the GenBank with accession numbers MZ491847, MZ702791-MZ702801 (Table S1).

Table 1

The quantity of the sequencing data and coverage depth of the 12 assembled plastomes.

\begin{tabular}{|llll|}
\hline Species & Raw reads no. & Mapped reads No. & coverage $(\mathbf{x})$ \\
\hline F. megaspartanium & $18,203,454$ & $1,917,714$ & 1810 \\
\hline F. cymosum & $20,895,137$ & $1,375,223$ & 1291 \\
\hline F. statice & $14,703,147$ & $1,655,635$ & 1551 \\
\hline F. leptopodum var. grossii & $6,778,507$ & 669,765 & 1132 \\
\hline F. leptopodum & $15,258,779$ & 836,663 & 818 \\
\hline F. urophyllum (lianas) & $17,566,621$ & 446,898 & 426 \\
\hline F. sp & $19,824,585$ & 939,432 & 906 \\
\hline F. urophyllum & $25,542,740$ & $1,012,882$ & 969 \\
\hline F. qiangcai & $15,396,608$ & $2,356,508$ & 2215 \\
\hline F. caudatum & $13,997,722$ & 167,898 & 681 \\
\hline F. gracilipes var. odontopterum & $12,750,680$ & $5,614,317$ & 5265 \\
\hline F. gracilipes & $20,659,431$ & 898,566 & 2716 \\
\hline
\end{tabular}

The obtained 12 Fagopyrum plastomes have a length in the range of 158,768 bp to 159, $985 \mathrm{bp}$. All the species included in this study possessed plastome with canonical quadripartite structure as well as two 
IR regions (30, 848-30, 729 bp) which separate the LSC (85, 051-84, $209 \mathrm{bp})$ and SSC (13, 348-13, 155 bp) regions. A representative plastome map of Fagopyrum was drawn using OGDRAW, as shown in Fig. 2. The GC contents of these 12 newly sequenced Fagopyrum plastomes ranged from $37.8-37.9 \%$. A total of 131 genes were annotated, and 86 protein-coding genes ( 72 single-copy genes and seven genes with two copies), 37 tRNA genes (19 single-copy genes and nine genes with two copies), and eight rRNA genes (four genes with two copies) were identified (Table 2). In Fagopyrum species, gene numbers and orders were found to be conserved, similar to those of many Polygonaceae genera (Table S3). The multiple sequence alignments in Fagopyrum were performed using mVISTA software that revealed the high sequence similarity (>90\%) of 12 newly sequenced plastomes (Fig. S1).

Table 2

Plastomes characteristics of newly sequenced Fagopyrum species.

\begin{tabular}{|c|c|c|c|c|c|c|c|}
\hline Taxon & Species & $\begin{array}{l}\text { Genome } \\
\text { (bp) }\end{array}$ & $\begin{array}{l}\text { LSC } \\
\text { (bp) }\end{array}$ & IR (bp) & $\begin{array}{l}\text { SSC } \\
\text { (bp) }\end{array}$ & $\begin{array}{l}\text { Gene } \\
\text { content }\end{array}$ & $\begin{array}{l}\text { GC } \\
\text { content(\%) }\end{array}$ \\
\hline \multirow{2}{*}{$\begin{array}{l}\text { cymosum } \\
\text { group }\end{array}$} & F. cymosum & 159,935 & 85,038 & 30,792 & 13,313 & $86 / 37 / 8$ & 37.9 \\
\hline & $\begin{array}{l}F \\
\text { megaspartanium }\end{array}$ & 159,985 & 85,051 & 30,793 & 13,348 & $86 / 37 / 8$ & 37.9 \\
\hline \multirow{10}{*}{$\begin{array}{l}\text { urophyllum } \\
\text { group }\end{array}$} & F. caudatum & 159,197 & 84,347 & 30,844 & 13,162 & $86 / 37 / 8$ & 37.8 \\
\hline & $\begin{array}{l}\text { F. gracilipes var. } \\
\text { odontopterum }\end{array}$ & 159,297 & 84,455 & 30,841 & 13,160 & $86 / 37 / 8$ & 37.8 \\
\hline & F. gracilipes & 159,097 & 84,209 & 30,848 & 13,192 & $86 / 37 / 8$ & 37.9 \\
\hline & F. leptopodum & 158,768 & 83,902 & 30,839 & 13,188 & $86 / 37 / 8$ & 37.9 \\
\hline & $\begin{array}{l}\text { F. leptopodum } \\
\text { var. grossii }\end{array}$ & 159,343 & 84,444 & 30,840 & 13,219 & $86 / 37 / 8$ & 37.8 \\
\hline & F. qiangcai & 159,144 & 84,531 & 30,729 & 13,155 & $86 / 37 / 8$ & 37.8 \\
\hline & F. $s p$ & 159,341 & 84,449 & 30,848 & 13,196 & $86 / 37 / 8$ & 37.9 \\
\hline & F. statice & 159,265 & 84,392 & 30,841 & 13,191 & $86 / 37 / 8$ & 37.8 \\
\hline & $\begin{array}{l}\text { F. urophyllum } \\
\text { (lianas) }\end{array}$ & 159,427 & 84,520 & 30,846 & 13,215 & $86 / 37 / 8$ & 37.8 \\
\hline & F. urophyllum & 159,288 & 84,425 & 30,846 & 13,171 & $86 / 37 / 8$ & 37.8 \\
\hline
\end{tabular}

LSC: Large single copy region; SSC: Small single copy region; IR: Inverted repeat region.

\section{Plastome size}

This study summarizes the complete sequence length and lengths of the four constituent regions (LSC, IRb, SSC, and IRa) from 69 plastomes of Polygonaceae species. To analyze the correlation between expansion/contraction of IR/SC of the plastomes and phylogeny, a length stacked bar chart was 
constructed according to the phylogenetic framework (Fig. 3, Table S4). The Fagopyrum clade was located at the base of the phylogenomic framework of Polygonaceae. The complete sequence of plastomes in Fagopyrum ranges from 158,768 bp (Fagopyrum leptopodum) to 159,985 bp (Fagopyrum megaspartanium). The LSC region was found located between $83,902-85,135 \mathrm{bp}$, with the IR region and the SSC region spanning from 30,685-30,870 bp and 13,093-13,348 bp, respectively (Fig. 3a, Table S4). The gene region was found between $114,081-114,406$ bp as well as an intergenic region was spanning between 44,678-45,598 bp (Fig. 3b, Table S4). In the Polygonaceae except for Fagopyrum, the species with the largest (170,974 bp) and smallest (158,981 bp) plastome sizes were Afrobrunnichia erecta and Persicaria chinensis respectively, both the species were located at the base of the phylogenomic framework. The length of LSC regions, IR region and SSC region is between 84,347-88,878 bp, 30,34834,631 bp and t 12,762-13,653 bp, respectively (Fig. 3a, Table S4). Furthermore, the length of gene region was between $113,671-117,858 \mathrm{bp}$, and the length of intergenic region was between $45,174-53,374 \mathrm{bp}$ (Fig. 3b, Table S4).

\section{IR/SC boundaries}

The IR/SC boundaries among 20 Fagopyrum plastomes and 49 plastomes of other genera in Polygonaceae were compared. In Polygonaceae, four types of IR/SC boundaries were summarized and presented in the phylogenetic tree (Fig. 4). The type I IR/SC boundary was found most common among the 12 newly obtained plastomes of Fagopyrum and in most genera of Polygonaceae, such as Calligonum, Muehlenbeckia, Oxyria, Persicaria, Rheum, and some species of Rumex. In this type of boundary, the rps19 and $n d h F$ genes straddled the LSC/IRb and IRb/SSC boundary regions respectively. The SSC/IRa boundary genes were $r p s 15$ and $y c f 1$, while $r p / 2$ and $t r n H$ were the IRa/LSC boundary genes. The type II IR/SC boundary was found in some species of Fagopyrum (F. esculentum, F. esculentum subsp. ancestrale, F. dibotry3, and F. luojishanense), similar to the type I boundary, except that the rps15 gene straddled the SSC/IRa boundary region. The type III IR/SC and type IV boundary were found in Fallopia and Afrobrunnichia, respectively. The type III and type IV IR/SC boundaries similar to the type I boundary, except that rps19 and rp/14 were present in the IRb region, and the IR region expanded to a greater degree in the type IV boundary compared with the type III boundary. A detailed comparison of the IR/SC boundaries has been presented in Fig S2, S3.

\section{Sequence diversity}

The single nucleotide polymorphisms (SNPs), insertions/deletions (indels), as well as phylogenetic informativeness $(\mathrm{Pi})$ of the 184 non-overlapping matrices of gene region and 173 non-overlapping matrices of the intergenic region were calculated based on multiple plastome sequences in Fagopyrum. The gene matrix with the highest $\mathrm{Pi}$ value was found in $c / p P$ gene regions, followed by $a c c D$ and $r p s 15$ genes. On the other hand, the intergenic matrix with the highest $\mathrm{Pi}$ value was observed in $p s b E$-petL gene region, followed by $r p / 32$-trnL and trnS-trnG genes (Fig. 5). The genes or intergenic regions with high $\mathrm{Pi}$ 
value matrices were identified as hotspots. The Pi value of the top 20 hotspots of gene regions ranged from 0.02617 to 0.05215 , whereas for the intergenic regions, it ranged from 0.05079 to 0.13248 (Table S5). The result of variation analysis showed that the LSC and SSC regions were more divergent than the two IR regions and had a higher divergence in the intergenic regions than gene regions. The top 20 hotspots of neither the gene regions nor the intergenic regions were found in the IR region (Fig. 5).

\section{Phylogenetic analysis}

Phylogenomic analyses based on the complete plastome sequences support the monophyly of Fagopyrum with two clades (urophyllum group and cymosum group) in the genus. A total of 69 complete plastome sequences of Polygonaceae and two plastomes of outgroup were used for the phylogenomic inference (Table S2). Phylogenomic tree constructed by the ML method, provides strong support for the monophyly of Fagopyrum (bootstrap support (BS) =100) (Fig. 4). The genus Fagopyrum clustered at the base of Polygonaceae as a sister clade with Calligonum, Fallopia, Oxyria, Rheum, and Rumex genera. In Fagopyrum, the phylogenomic relationships inferred from the BI, ML, and MP analyses were consistent (Fig. 6), and the tree showed the formation of two monophyletic clades, i.e., the cymosum group and the urophyllum group, with strong support [BS $=100$, posterior probabilities $(P P)=1]$. Seven subclades were recovered within the Fagopyrum, including(I) F. leptopodum, F. leptopodum var. grossii, and F. statice; (II) F. gracilipes, F. gracilipes var. odontopterum, and F. Iuojishanense, (III) F. urophyllum (lianas) and F. sp.; (IV) F. caudatum and F. qiangcai, (V) F. megaspartanium, F. cymosum, F. dibotrys1, F. dibotrys2, and F. dibotrys3; (VI) F. tataricum and F. tataricum (cultivar Miqiao); (VII) F. esculentum and F. esculentum subsp. ancestrale. The subclades I-IV belonged to the urophyllum group clade, while subclades V-VII belonged to the cymosum group clade.

To identify the useful molecular markers, six data matrices of Fagopyrum plastome sequences were used to construct phylogenies. The results demonstrated that the tree topologies generated based on matrices consisting of all gene regions, 20 gene regions, and eight gene regions were consistent with the tree, constructed based on the complete plastome sequences. The topological structure of the subclade of $F$. cymosum and $F$. megaspartanium showed a difference between the trees based on complete plastome sequences and matrices of all intergenic regions, 20 intergenic regions, and eight intergenic regions. Similarly, the topological structure of the subclade of F. caudatum and F. qiangcai and the subclade of $F$. gracilipes revealed the difference between the trees based on complete plastome sequences and matrices of eight intergenic regions (Fig. S4, Table S5).

\section{Repeats}

Short dispersed repeats (SDRs) (including forward repeats, reverse repeats, complement repeats, and palindromic repeats), simple sequence repeats (SSRs) as well as tandem repeats were analyzed in this study (Fig. 7). The results of plastome SDRs analysis showed considerable variations in the numbers and length of repeats in different Fagopyrum groups. The plastomes with the most numbers of SDRs $(F$. 
megaspartanium) and with the least number of SDRs ( $F$. esculentum subsp. ancestrale) were found in the cymosum group (Fig. 7a). Most of the SDRs were found as forward repeats followed by reverse repeats. The forward and reverse repeats exist in each plastome of Fagopyrum, complementing the existing repeats in seven plastomes of Fagopyrum. The palindromic repeats exist only in plastomes of $F$. qiangcai, F. leptopodum, and F. megaspartanium (Fig. 7b).

The number of SSRs found in the plastomes of the cymosum group, and urophyllum group was 144-159 and 162-169, respectively. Most of the SSRs were mono-nucleotide repeats (98.32\%) having the bases of mono-nucleotide repeat sequences adenine/thymine (97.63\%) (Fig. 7c). The number of tandem repeats in plastomes for the cymosum group and urophyllum groups was 9-22 and 12-17, respectively. There were obvious differences between the cymosum and urophyllum groups in terms of the number of SSRs, SDRs, or tandem repeats (Fig. 7d).

\section{Plastome sequence similarity}

The comparison of plastome revealed the high sequence similarity in groups among Fagopyrum (Fig. 8). Among the cymosum group, the sequence identity of plastome was found from $97.009-99.748 \%$, whereas in the urophyllum group, it ranges from $98.218-99.690 \%$. On the other hand, the sequence identity of plastome between cymosum and urophyllum group was from 91.238-91.829\%. Moreover, the sequence identity of plastome gene regions in the cymosum group ranged from $98.563-99.924 \%$, and the urophyllum group ranged from $99.118-99.900 \%$. The plastome gene regions between the cymosum and urophyllum groups showed sequence identity from 96.055-96.353\% (Fig. 8).

\section{Discussion}

\section{Plastome variation}

The comparative analysis of plastome sequences provides new insights into sequence variation and molecular evolutionary patterns $[27,40]$. The current study found conserved patterns among plastomes of Fagopyrum were conserved in the quadripartite structure, length, gene order, and GC content (Fig. S2, Table 2, S3). Although the results were found similar to previous studies that explored a few species of Fagopyrum [36-37], the evolutionary dynamics of Fagopyrum plastomes in the Polygonaceae is not well understood. A detailed plastome characteristics comparison between Fagopyrum with other Polygonaceae genera was performed, analyzing the correlation between plastome variations with phylogeny. The phylogenetic framework was constructed with genome size and a phylogenetic tree with IR/SC boundary type (Fig. 3, 4). These results revealed the variations of the Fagopyrum plastomes that are mainly reflected in intergenic regions length and SSC/IRa boundary.

IR contraction and expansion are common evolutionary phenomena and may cause variations in the plastome size [38-39]. Moreover, the loss or insertion of the gene also influences the plastome size [41]. In the plastomes of Polygonaceae, the IR region was found more conservative than the SC region, 
especially LSC, as most of the plastome size variations were in the LSC. Although most of the species (including Fagopyrum species) in Polygonaceae don't have IR expansion and gene duplication (Fig. 4), the IR expansion occurred only in a few species of Polygonaceae, i.e., Afrobrunnichia erecta, and Fallopia sachalinensis. The Fagopyrum has a smaller plastome size (158,768-159,985 bp) in comparison with most of the other genera (158,981-170,232 bp) (Fig. 3a, Table S4). The IR expansion with gene duplication of rps19 in Fallopia sachalinensis and genes duplication of rps19, rp/22, rps3, rp/16, rp/14 in Afrobrunnichia erecta caused only $196 \mathrm{bp}$ and $3574 \mathrm{bp}$ plastome sequences to increase, respectively (Fig. 4, Fig S3). However, this increased length does not explain the difference in sequence length between Fagopyrum plastomes (3500-4717 bp, 10,247-11,464 bp) (Table S4). The plastomes that lack IR expansion and gene insertion, the length difference, such as between Atraphaxis irtyschensis and Fagopyrum species, reached 4207-5424 bp (Fig. 4, Table S4). Hence, the smaller plastome size of Fagopyrum may have been caused by factors other than IR contraction or gene loss.

In general, the short insertion/deletions (indels) lead to length variation in the plastome sequence. Although these variations occur in both the gene region and the intergenic region, they were found more in the intergenic region than gene region [42]. In the plastomes of Polygonaceae, the number of genes, as well as the length of gene regions, are mostly similar (Table 2, Fig. 3b). The length variation in the plastome sequences was evident in intergenic regions (Fig. $3 \mathrm{~b}$ ), as well as and the trend of length variation was very similar to the LSC as well as the complete plastome sequence (Fig. 3a). The results of sequence comparison among Fagopyrum plastomes showed a more frequent appearance of indels in the intergenic regions than the gene regions (Fig. 5). In Polygonaceae, the plastome intergenic region length was calculated for species. The intergenic region of Fagopyrum (44,678-45,598 bp) was found shorter than most of the other genera without IR expansion (45,174-49,747 bp). Taking the maximum difference value as an example, the longest intergenic region difference (5069 bp) between Fagopyrum species (Fagopyrum leptopodum) and other genera species (Atraphaxis irtyschensis) accounted for most of the maximum plastome size difference (5424) between them (Table S4). These results suggest that the plastome size variation is caused by indels in intergenic regions. Moreover, the small plastomes size of the Fagopyrum is due to short intergenic regions with fewer insertions or more deletions. Thus, it may be the main reason for the variation in plastome size of other Polygonaceae taxa other than IR expansion with gene duplication.

According to the previous reports, the plastomes of Polygonaceae have a typical quadripartite structure (Rumex) [43], (Calligonum) [44], (Rheum) [45]. In this study, a comparison of plastomes between Fagopyrum and other genera of Polygonaceae revealed the existence of similar structure also in Fagopyrum as well as the difference in the boundaries of IR/SC (Fig. 4). Logacheva et al. [46] reported that $F$. esculentum differed from most other flowering plants at the junctions of the SSC/IRa boundary. Although, the ycf1 gene straddled the SSC/IRa boundary in most flowering plants, the rps 15 gene straddled in the plastome of $F$. esculentum in a similar region. The rps 15 gene straddled the SSC/IRa boundary not only in $F$. esculentum but also in the plastome of $F$. esculentum subsp. ancestrale, $F$. dibotry 3 , and F. luojishanense (Fig. 4 type II). This boundary variation occurs due to the different location of the initiation codon of the rps 15 gene from other plastomes of Fagopyrum. In most Fagopyrum 
plastomes, the rps15 gene has an initiation codon in SSC, while in its variation, it was found in IRa. However, this variation nested distribution in different groups of Fagopyrum and is not a common characteristic of Fagopyrum plastomes. Consequently, this variation cannot represent the plastome evolutionary position of Fagopyrum in Polygonaceae due to lacking boundary at the genus level. It could be deemed that those species which have type II boundary have evolved more than other species in Fagopyrum. This phenomenon may be caused by the fact that sequences adjacent to the IR/SC boundaries are more susceptible to mutations. In general, the DNA sequence at the boundary have sufficient variation to be used as molecular markers in many taxa, for example ycf 1 gene and intergenic region trnH-psbA, which is adjacent to the SSC/IRa boundary and IRa/LSC boundary, respectively [47]. The sequence diversity analysis of Fagopyrum plastomes revealed that the rps 15 gene did exhibit the highest sequence diversity in the gene region (Table S5). Thus, it can be used as candidate molecular markers to identify some species of Fagopyrum.

The evolutionary patterns and species relationships can be considered by the nested distribution of structural variations [40,48]. The type III and IV IR/SC boundaries occurred in Afrobrunnichia erecta and Fallopia sachalinensis, respectively as well as their variations were caused by expansion of IRa. The IRa/LSC boundary of these two species includes an extra sequence in addition to homologous sequences of plastomes in Polygonaceae. Accordingly, the part of the LSC sequence corresponding to IRa expansion is divided into IRb region (including $r p s 19, r p / 22, r p s 3, r p / 16, r p / 14$ and their intergenic sequences in Afrobrunnichia erecta plastome as well as rps19 sequences in Fallopia sachalinensis) (Fig. 4). The phylogenetic tree revealed the distally branching clades to be less conserved than relative basally branching clades [48]. So, it can be inferred that the IR expansion of Afrobrunnichia erecta and Fallopia sachalinensis plastome is caused by the retention of common ancestral characteristics, and gene loss may have occurred in most species of Polygonaceae. However, the inference is based on the comparison of a single plastome of Afrobrunnichia and Fallopia with other Polygonaceae plastomes. Therefore, future research with more samples is needed to determine whether the gene duplication of the IR region is common in Afrobrunnichia and Fallopia.

\section{Phylogenomic inferences and species relationships}

A well-supported phylogenomic framework was reconstructed based on 12 newly sampled plastomes included in the present study as well as eight plastomes reported in previous studies [35-36, 49-50]. The monophyly and group division based on DNA loci was also found consistent with previous studies in Fagopyrum [12, 15-17, 25, 51-52]. The urophyllum group consisted the three subgroups, including $F$. leptopodum-F. statice subgroup, F. capillatum-F. gracilipes subgroup, and F. callianthum-F. pleioramosum as reported in the previous studies. However, the relationship among these subclades has been inconsistent in different studies, and new species were rarely included. The current study resolves the uncertain systematic position of these subgroups. The subclade I (F. leptopodum, F. leptopodum var. grossii, and F. statice) was found closely related to the subclade II (F. gracilipes, F. gracilipes var. odontopterum, and $F$. luojishanense) followed by the non-monophyletic species $F$. urophyllum ( $F$. urophyllum (liana), F. sp, and F. urophyllum) and subclade IV (F. caudatum and F. qiangcai) (Fig. 6). Additionally, this study also provides insights into the new species described in recent years. The 
subclade II includes a recently described species, i.e., F. luojishanense [23], which was morphologically most similar to $F$. gracilipes. A recent study based on the DNA loci suggested that $F$. luojishanense doesn't belong to the cymosum group [36]. However, due to the lack of plastome data on members of the urophyllum group, the systematic position of $F$. luojishanense remains unclear. Our phylogenetic tree indicated that $F$. Iuojishanense belonged to the urophyllum group and was closely related to $F$. gracilipes (Fig. 6).

The subclade IV included $F$. caudatum, as well as the newly described $F$. qiangcai, which has been considered closely related to $F$. esculentum based on morphology [22] or to F. leptopodum based on molecular data [12]. In this study, F. qiangcai was taken as a sample from a population with leafy bases, leaves with bright red veins, and white punctate adaxial leaf surfaces from Lixian, Sichuan Province (Fig. 1g). The morphological characteristics of samples were also similar to the F. callianthum, which was reported by Ohsako and Ohnishi [19]. So, the F. qiangcai was considered as a synonym of $F$. callianthum, likely to report of Tang et al. [3]. Another sample F. caudatum was taken from the subclade IV. It has two other synonyms, namely $F$. pleioramosum and $F$. wenchuanense [3]. Based on both plastome and nuclear DNA loci, $F$. pleioramosum showed more closeness to $F$. qiangcai ( $F$. callianthum) $[15-16,18-20,53]$. On the other hand, $F$. wenchuanense and $F$. cymosum were found closely related to $F$. gracilipes, based on the morphology [22] and molecular data [12], respectively. The previous phylogenetic analyses were unable to resolve the relationship between $F$. caudatum ( $F$. pleioramosum/F. wenchuanense) and other species based on a small number of DNA loci. Our findings supported the close relationship between $F$. caudatum (F. pleioramosum/F. wenchuanense) and F. qiangcai (F. callianthum) and confirmed the systematic position of this subclade at the base of urophyllum group (Fig. 6 ). The subclade IV, including F. caudatum and $F$. qiangcai, clustered as an independent branch in the urophyllum group but was not closely related to the species $F$. leptopodum and F. gracilipes like previous reports [12, 22]. Moreover, we propose to rename the $F$. callianthum- $F$. pleioramosum subgroup to the $F$. caudatum-F. qiangcai subgroup, which seems more appropriate and includes a species name from Flora of China [1].

The plastome phylogenomic provided strong support for relationships between subclades in the cymosum group. The reconstructed subclade $V$ (F. megaspartanium, F. cymosum, F. dibotrys1, F. dibotrys 2 , and $F$. dibotrys 3 ) was found more closely related to subclade VI (F. tataricum and $F$. tataricum (cultivar Miqiao) than subclade VII (F. esculentum and F. esculentum subsp. ancestrale) (Fig. 6), like findings of previous studies $[4,15-17]$. However, the taxonomic status of $F$. megaspartanium and $F$. cymosum is still a controversial issue.

In addition, F. megaspartanium was treated as a variety or a closely related species of F. cymosum [17, 54]. According to Ohsako and Li [4], in a phylogenetic analysis based on matK sequences, F. cymosum appeared to be a complex species with two branches. The report of Chen [55] suggests that $F$. megaspartanium might be an ancestor of the cultivated buckwheat species viz., $F$. esculentum, and $F$. tataricum. Furthermore, it was suggested that the $F$. esculentum subsp. ancestrale might be a hybrid species between F. cymosum and F. esculentum [55], and there is a complex evolutionary history among the F. cymosum species. 
The phylogenetic analysis based on plastome sequences strongly supported the two branches of the complex species F. cymosum, i.e., one branch including our samples F. cymosum, F. dibotrys1, and $F$. megaspartanium, while the other comprised of the samples F. dibotrys2 and F. dibotrys3 (Fig. 6). In contrast, the topology of branches based on a few intergenic region sequences differed from that of trees based on plastome sequences (Fig. S4). The topologies supported by poor posterior probabilities and bootstrap support may lead to misunderstanding the species relationships. Therefore, we speculate that F. megaspartanium and $F$. cymosum belong to the same branch of the $F$. cymosum species complex, and F. megaspartanium is the representative of this branch based on the phylogenomic tree of this study. Furthermore, to clarify the other branches of the F. cymosum species complex, further sampling of the subclades $V$ is needed in future studies.

The phylogenetic framework based on plastomes not only elucidated the taxonomic relationships but also enhanced our understanding of morphological characteristics of Fagopyrum. Achene size is considered one of the key taxonomic characteristics of Fagopyrum [2]. In this study, the achene size show difference between the urophyllum group and cymosum group in the phylogenetic tree. Most of the achenes of species in the urophyllum group are less than $5 \mathrm{~mm}$ and gradually become smaller from the basal branch (F. caudatum-F. qiangcai subclade) to the distal branch ( $F$. leptopodum-F. statice subclade). On the contrary, in the cymosum group, the achenes of species are mostly greater than $5 \mathrm{~mm}$ and gradually increase from the basal branch ( $F$. esculentum subclade) to the distal branch ( $F$. megaspartanium-F. cymosum subclade) (Fig. 6). The results suggest that these two groups are likely to have a common ancestor with an achene size of around $5 \mathrm{~mm}$ and evolved in different directions, resulting in the present achene size differences. Thus, based on phylogenetic trees, it can be inferred that the evolution of morphological characters facilitates classification and species identification.

Moreover, detecting the highly informative and variable genome regions can be important for diagnostic genetic marker development and DNA barcoding [27]. In Fagopyrum, plastome regions $r b c L$, accD, trnK (UUU), trnC (GCA)-rpoB, matK, trnH-psbA, trnL, psbE-psbL, and $n d h A$ intron have been widely used to construct the phylogeny at the species level, which is often supplemented with nrDNA ITS and/or nuclear gene sequences ( $F L O / L F Y, A G)[12,15-17,19,53,57]$. In general, there is a greater variation in intergenic regions than in gene regions, as has also been observed in this study (Table S5). Although to increase the sequence variation and obtain considerable support, some intergenic regions have also been used for phylogenetic analysis. The rapid rate of evolution of intergenic regions may lead to topology confusion. This study demonstrates that phylogenetic analysis based on gene regions concatenation is more comparable to the complete plastome than intergenic regions concatenation (Fig. S4). It shows that the $\mathrm{Pi}$ of the gene regions is more appropriate for species identification than intergenic regions. Therefore, to reconstruct the phylogenetic tree of Fagopyrum, the combination of the top eight gene regions (rps15, trnK, trnL, matK, ndhA, clpP, rpoC2, rp/16) were considered as an economical and accurate candidate marker.

To explore the species relationship of Fagopyrum, in addition to DNA loci and their combinations, repeats or SNPs were also be used as molecular markers [58-59]. The number of different types of repeat 
sequences in Fagopyrum plastomes differs in different groups (Fig. 7), as well as similarity of plastomes also differed significantly between intra-groups and inter-groups (Fig. 8). These results suggest that sequence diversity also reveals either distant or close relationships between species. However, unfortunately, these differences were not found significant between subgroups. Thus, based on the mutation hotspot and the complete plastome sequence, phylogenetic analysis is still the best way to determine the relationship between Fagopyrum species.

\section{Conclusions}

This study provides a detailed comparison of plastome characteristics in Fagopyrum and a phylogenomic framework of Fagopyrum with strongly support. The plastome size difference, which is mainly due to deletions of intergenic regions, shows that the Fagopyrum plastome has been conserved within genera and remains specific between genera. The IR/SC boundary variations, which mainly occur due to gene loss, revealed the evolutionary dynamics of Fagopyrum in Polygonaceae. In Fagopyrum, the plastome sequence diversification demonstrates its power in resolving evolutionary relationships. It is possible to reconstruct phylogenetic relationships with high support even from several gene sequences. Moreover, the number of repeats in plastomes and relatively low sequence similarity between groups of Fagopyrum allows the development of molecular markers for species identification based on SNPs and repeats. As demonstrated in this study, plastome sequences are capable of revealing species relationships and evolutionary changes in taxa. In addition to appending new genomic resources, these findings will be useful for future studies of the evolution and phylogeny of Fagopyrum.

\section{Methods}

\section{Taxon sampling and DNA sequencing}

A total of 12 wild individuals representing the eight species and two varieties were collected and sequenced (Fig. 1, Table S1). The formal identification of plants was performed according to the Flora of China [1]. The names of the species, locations of the specimen collections, voucher numbers, and GenBank accession numbers for all samples used in this study are listed in Table S1. All the samples were identified by An-hu Wang and Qing-fu Chen (Panxi Crops Research and Utilization Key Laboratory of Sichuan Province, Xichang University and Research Center of Buckwheat Industry Technology, Guizhou Normal University) based on the morphological characters and the species were preserved in the herbarium of Panxi Crops Research and Utilization Key Laboratory of Sichuan Province. The fresh leaf samples were collected and dried using silica gel. To extract the total DNA from collected samples, a modified Cetyltrimethylammonium Bromide (CTAB) method was used [60]. The sequencing was performed on the Illumina NovaSeq 6000 platform at TSINGKE Biological Technology Co., Ltd. (Beijing, China) using the paired-end $150 \mathrm{bp}$ reads with an average insert size of 300-400 bp. Additionally, 57 plastomes of Polygonaceae (including eight plastomes of Fagopyrum) and two plastomes of Plumbaginaceae were downloaded for the phylogenetic analysis and sequence comparison. The GenBank accession numbers for taxa sampled in this study are listed in Table $\mathbf{S 2}$. 


\section{Plastome assembly and annotation}

The quality check of raw reads was performed using FastQC v0.11.9. The plastomes were de novo assembled using GetOrganelle v1.6.2 [61] considering the plastomes of $F$. dibotrys (KY275181) and $F$. Iuojishanense (KY275182) as references. To verify the sequencing depth and overlapping contigs, the cleaned reads were mapped to the reference plastomes using Geneious R11.0.5 [62] (Table 1). The Plastid Genome Annotator (PGA) was used to annotate the plastomes [63], followed by the validation of annotation using GeSeq [64] (Table 2). A circular map of the plastomes was generated employing the Organellar Genome DRAW (OGDRAW) [65] (Fig. 2).

\section{Plastome comparative analysis}

The guanine-cytosine content (GC content) of each accession was determined in Geneious. Since the large single-copy (LSC) and small single-copy (SSC) regions were flanked by the two inverted repeat (IR) regions, the junctions between these regions were found by calculating the IR region length using REPuter [66] (Fig. S2, S3). Fagopyrum plastomes were characterized using the Multiple Alignment using Fast Fourier Transform (MAFFT) algorithm [67], and the checked sequence similarity was assessed in Geneious (Fig. 3, 8, Table S4). The sequence divergence among 12 plastomes was visualized using the mVISTA program [68], considering F. gracilipes as a reference (Fig. S1). The sequence variation of Fagopyrum plastomes was mapped by dividing the gene sequences and the intergenic sequences without IRa regions into overlapping and non-overlapping matrices of 500 and 250 bp, respectively. To calculate the phylogenetic informativeness ( $\mathrm{Pi})$, insertions/deletions (indels), and single nucleotide polymorphisms (SNPs), DnaSP was employed [69] (Fig. 5).

\section{Repeats analysis}

Using the online program REPuter [66], SDRs were identified, including four types, i.e., forward repeats, reverse repeats, complement repeats, and palindromic repeats (Fig. 7a, 7b). The parameters were set as (1) Hamming distance to 3; (2) $90 \%$ or greater sequence identity; and (3) minimal repeat size of $30 \mathrm{bp}$. SSRs were also identified via Perl script MISA [70], including mono, di, tri, tetra, penta, and hexanucleotides. The minimum numbers of the SSRs were set to 10, 5, 4, 3, 3, and 3 for mono, di, tri, tetra, penta, and hexanucleotides, respectively (Fig. 7c). An online program, Tandem Repeats Finder [71], was employed to find the tandem repeats, where the similarity percentage of two repeat copies was at least $90 \%$ as well as the minimal repeat size was 10 bp (Fig. 7 d).

\section{Phylogenetic analysis and hotspots identification}

The complete plastome sequences were used for phylogenetic studies of Polygonaceae and Fagopyrum. To investigate the systematic position of Fagopyrum in the family Polygonaceae, 69 plastome data sets 
encompassing a wide phylogenetic diversity in Polygonaceae were included in analyses. Limonium sinense and Plumbago auriculata from the family Plumbaginaceae were used to root the phylogenetic tree (Fig. 4). To investigate relationships among the species within the genus Fagopyrum, the phylogenetic analyses were performed using 20 plastomes. Afrobrunnichia erecta and Persicaria chinensis from the family Polygonaceae were used for rooting the phylogenetic tree (Fig. 6). Phylogenetic inference of Polygonaceae was conducted using the maximum likelihood (ML) method. Moreover, phylogenetic inference of Fagopyrum was conducted using three approaches, including ML, maximum parsimony (MP), and Bayesian inference (BI).

The ML analysis was performed using RAxMLHPC2 v8.0 [72] on the Cyberlnfrastructure for Phylogenetic Research (CIPRES) Science Gateway v3.3 [73]. The analysis of 1,000 rapid bootstrap replicates (-x) was followed by a search for the best-scoring ML tree in one single program run (- $f a)$. The MP analysis was carried out in PAUP* v4.0 b10 [74] with equally weighted and unordered characters. Searches were performed on 100 replicates of random taxon addition using tree-bisection-reconnection (TBR) branch swapping with the MulTrees option. Bootstrap analyses, including parsimony bootstrap percentages (PBP), and 1,000 pseudoreplicates, were carried out with the same parameters to examine the relative level of clade support. BI analyses were performed using MrBayes v3.2 [75]. Tree searches with a randomly chosen starting tree were run for each dataset consisting of one million generations, with sampling every 100 generations. An initial $25 \%$ of sampled trees were discarded. The posterior probability of values was calculated from the remaining trees. Stationarity was reached when the average standard deviation of split frequencies was below 0.01 .

To explore the informative regions of the plastome, six datasets consisting of the following sequences were included in phylogenetic analyses using three different methods, namely ML, MP, and BI: (a) all gene regions; (b) all intergenic regions; (c) the top 20 hotspots of gene regions; (d) the top 20 hotspots of intergenic regions; (e) the top eight hotspots of gene regions; and (f) the top eight hotspots of intergenic regions (Fig. S4, Table S5).

\section{Declarations}

\section{Ethics approval and consent to participate}

We comply with the IUCN Policy Statement on Research Involving Species at Risk of Extinction and the Convention on the Trade in Endangered Species of Wild Fauna and Flora and confirm that all methods were performed in accordance with the relevant guidelines/regulations/legislation. These wild species samples in this study have not been included in the list of key protected plants and the sampling of wild species in this study was met local policy requirements.

\section{Consent for publication}

Not applicable. 
Availability of data and materials

All sequences used in this study are available from the National Center for Biotechnology Information (NCBI) (see Additional file 1: Table S1) and also from Supplementary Information (Additional file 2: Plastome DNA sequences of Fagopyrum). The GenBank accession numbers are MZ491847 and MZ702791-MZ702801. All sequences will be made public after this manuscript is published or after July 2, 2023.

\section{Competing interests}

The authors declare no conflicts of interest.

\section{Funding}

This work was supported by the Fundamental Research Funds for the Central Universities (2020SCUD003) and the Open Project Program of Panxi Crops Research and Utilization Key Laboratory of Sichuan Province (No.SZKF2105).

\section{Author contributions}

Yi Yang: Conceptualization, Methodology; Qiu-jie Li, Yu Liu, An-hu Wang \& Jian-mei Wang: Field investigation, Materials collection; An-hu Wang \& Qing-fu Chen: Identifying material; Qiu-jie Li, Yu Liu \& Lu Peng: Data curation, Analysis, Visualization; Qiu-jie Li \& Lu Peng: Writing-Original draft preparation; Qiu-jie Li \& Yi Yang: Writing-Reviewing and Editing; Yi Yang: Supervision. All authors have read and approved the manuscript.

\section{Acknowledgements}

We are very grateful to Xinghui Wu and Qizong Cai of Guizhou Normal University for assistance with sample collection.

\section{References}

1. Li A, Hong S. Fagopyrum Miller, In: Wu, ZY, editors. Flora of China. Beijing: Science Press; 2003. p. 320-323.

2. Chen QF, Huang XY, Li HY, Li J, Cui YS. Recent progress in perennial buckwheat development. Sustainability. 2018;10:536.

3. Tang Y, Shao JR, Zhou, ML. A Taxonomic Revision of Fagopyrum Mill from China. Plant Genet Resour. 2019;20:646-653.

4. Ohsako T, Li CY. Classification and systematics of the Fagopyrum species. Breed Sci. 2020;70:93100. 
5. Zhang KX, Fan Y, Weng WF, Tang Y, Zhou ML. Fagopyrum longistylum (Polygonaceae), a new species from Sichuan, China. Phytotaxa. 2021;482:173-182.

6. Suvorova G, Zhou ZL. Distribution of cultivated buckwheat resources in the world. In: Zhou ML, editors. Buckwheat Germplasm in the world. San Diego: ELSEVIER, London: Academic Press; 2018. p. 9-20.

7. Wijngaard HH, Arendt EK. Buckwheat. Cereal Chem. 2006;83:391-401.

8. Nina F, Janko R, Jože KI, Wang Z, Zhang Z, Ivan K. Tartary buckwheat (Fagopyrum tataricum Gaertn.) as a source of dietary rutin and quercitrin. J. Agric Food Chem. 2003;51:6452-6455.

9. Chinese Pharmacopoeia Commission. Fagopyri Dibotryis Rhizoma. In: Pharmacopoeia of People's Republic of China. Beijing: China Medical Science Press; 2015. p. 5:218.

10. Yasui Y, Hirakawa H, Ueno M., Matsui K., Katsube-Tanaka T, Yang SJ, et al. Assembly of the draft genome of buckwheat and its applications in identifying agronomically useful genes. DNA Res. 2016;23:215-224.

11. Zhang KX, He M., Fan Y., Zhao H., Gao B., Yang KL, et al. Resequencing of global Tartary buckwheat accessions reveals multiple domestication events and key loci associated with agronomic traits. Genome Biol. 2020;22:23.

12. Zhou ML, Wang, CL, Wang DZ, Zheng YD, Li FL, Zhu XM, et al. Phylogenetic relationship of four new species related to southwestern Sichuan Fagopyrum based on morphological and molecular characterization. Biochem Syst Ecol. 2014;57:403-409.

13. Gross MH. Remarquessur les Polygoneas de l'Asie Orientale. Bull Torrey Bot Club. 1913;23:7-32.

14. Roberty G, Vautier S. Les genres de Polygonacées. Boissiera. 1964;10:7-128.

15. Yasui Y, Ohnishi O. Phylogenetic relationships among Fagopyrum species revealed by the nucleotide sequences of the ITS region of the unclear rRNA gene. Genes Genet Syst. 1998;73:201-210.

16. Yasui Y, Ohnishi O. Interspecific relationships in Fagopyrum (Polygonaceae) revealed by the nucleotide sequences of the $r b c L$ and $a c c D$ genes and their intergenic region. Am $\mathrm{J}$ Bot. 1998;85:1134-1142.

17. HuangY, Li ZQ, Wang CL, Zou CY, Wen W, Shao JR, et al. psbE-psbL and $n d h A$ Intron, The Promising Plastid DNA barcode of Fagopyrum. Int J Mol Sci. 2019;20:3455.

18. Ohsako T, Ohnishi O. New Fagopyrum species revealed by morphological. Genes Genet Syst. 1998;73:85-94.

19. Ohsako T, Ohnishi O. Intra- and interspecific phylogeny of wild Fagopyrum (Polygonaceae) species based on nucleotide sequences of noncoding regions in chloroplast DNA. Am J Bot. 2000;87:573582.

20. Ohsako T, Yamane K, Ohnishi O. Two new Fagopyrum (Polygonaceae) species, F. gracilipedoides and F. jinshaense from Yunnan, China. Genes Genet Syst. 2002;77:99-408.

21. Tang Y, Zhou, ML, Bai DQ, Shao JR, Zhu XM, Wang DZ, et al. Fagopyrum pugense (Polygonaceae), a new species from Sichuan, China. Novon. 2010;20:239-242. 
22. Shao JR, Zhou ML, Zhu XM, Wang DZ, Bai DQ. Fagopyrum wenchuanense and Fagopyrum qiangcai, two new species of Polygonaceae from Sichuan, China. Novon. 2011;21:256-261.

23. Hou LL, Zhou ML, Zhang Q, Qi LP, Yang XB, Tang Y, et al. Fagopyrum luojishanense, a New Species of Polygonaceae from Sichuan, China. Novon. 2015;24:22-26.

24. Zhou ML, Zhang Q, Zheng YD, Tang Y, Li FL, Zhu XM, et al. Fagopyrum hailuogouense (Polygonaceae), one new species from Sichuan, China. Novon. 2015;24:222-224.

25. Jin JW, Li DR, Chen SF, Li B. A common Bistorta was misidentified as a novel species in Fagopyrum (Polygonaceae): the confirmation of the taxonomic identify of $F$. hailuogouense by morphological and molecular evidences. Phytotaxa. 2018;348:221.

26. Zhang YJ, Li DZ. Advances in phylogenomics based on complete chloroplast genomes. PI Divers Resour. 2011;33:365-375.

27. Gitzendanner MA, Soltis PS, Yi TS, Li DZ, Soltis DE. Plastome phylogenetics: 30 years of inferences into plant evolution. Adv Bot Res. 2018;85:293-313.

28. Li HT, Yi TS, Gao LM, Ma PF, Li DZ. Origin of angiosperms and the puzzle of the Jurassic gap. Nat Plants. 2019;5:461-470.

29. Ma Q, Du YJ, Chen N, Zhang LY, Li JH, Fu CX. Phylogeography of Davidia involucrata (Davidiaceae) inferred from cpDNA haplotypes and nSSR data. Syst Bot. 2015;40:796-810.

30. Zeb U, Dong WL, Zhang TT, Wang RN, Shahzad K, Ma X F, et al. Comparative plastid genomics of Pinus species: insights into sequence variations and phylogenetic relationships. J Syst Evol. 2019;58:118-132.

31. Liu F, Fan WS, Yang JB, Xiang CL, Mower JP, Li DZ, et al. Episodic and GC-biased bursts of intragenomic and interspecific synonymous divergence in Ajugoideae (Llamiaceae) mitogenomes. New Phytol. 2020;228:1107-1114.

32. Maier RM, Neckermann Kai, Igloi GL, Kossel H. Complete sequence of the maize chloroplast genome: gene content, hotspots of divergence and fine tuning of genetic information by transcript editing. $J$ Mol Biol. 1995;251:614-628.

33. Kim GB, Lim CE, Kim JS, Kim K, Lee JH, Yu HJ, et al. Comparative chloroplast genome analysis of Artemisia (Asteraceae) in East Asia: insights into evolutionary divergence and phylogenomic implications. BMC Genom. 2020;21:415.

34. Zhong QY, Fu XG, Zhang TT, Zhou T, Yue M, Liu JN, et al. Phylogeny and evolution of chloroplast tRNAs in Adoxaceae. Ecol. Evol. 2020;11:1294-1309.

35. Cho KS, Yun BK, Yoon YH, Hong SY, Mekapogu M, Kim KH, et al. Complete chloroplast genome sequence of tartary buckwheat (Fagopyrum tataricum) and comparative analysis with common buckwheat (F. esculentum). PLoS One. 2015;10:e0125332.

36. Wang CL, Ding MQ, Zou CY, Zhu XM, Tang Y, Zhou ML, et al. Comparative analysis of four buckwheat species based on morphology and complete chloroplast genome sequences. Sci Rep. 2017;7:6514. 
37. Yang BB, Li LD., Liu JQ, Zhang LH. Plastome and phylogenetic relationship of the woody buckwheat Fagopyrum tibeticum in the Qinghai-Tibet plateau. Plant Diversity. 2020;43:198-205.

38. Zhu A, Guo W, Gupta S, Fan W, Mower JP. Evolutionary dynamics of the plastid inverted repeat: the effects of expansion, contraction, and loss on substitution rates. New Phytol. 2016;209:1747-1756.

39. Weng ML, Ruhlman TA, Jansen RK. Expansion of inverted repeat does not decrease substitution rates in Pelargonium plastid genomes. New Phytol. 2017;214:842-851.

40. Yao G, Jin JJ, Li HT, Yang JB, Mandala VS, Croley M, et al. Plastid phylogenomic insights into the evolution of Caryophyllales. Mol Phylogenet Evol. 2019;134:74-86.

41. Guo YY, Yang JX, Bai MZ, Zhang GQ, Liu ZJ. The chloroplast genome evolution of Venus slipper (Paphiopedilum): IR expansion, SSC contraction, and highly rearranged SSC regions. BMC Plant Biol. 2021;21:248.

42. Li QJ, Su N, Tong RC, Zhang XH, Wang JR, Chang ZY, er al. Chloroplast genomes elucidate diversity, phylogeny, and taxonomy of Pulsatilla (Ranunculaceae). Sci Rep. 2020;10:19781.

43. Gui L, Jiang S, Wang H, Nong D, Liu Y. Characterization of the complete chloroplast genome of sorrel (Rumex acetosa). Mitochondrial DNA B. 2018;3:904-906.

44. Song F, Li T, Burgess KS, Feng Y, Ge XJ. Complete plastome sequencing resolves taxonomic relationships among species of Calligonum L. (Polygonaceae) in China. BMC Plant Biol. 2020;20:261.

45. Zhou T, Zhu H, Wang J, Xu Y, Wang X. Complete chloroplast genome sequence determination of rheum species and comparative chloroplast genomics for the members of Rumiceae. Plant Cell Rep. 2020;39:811-824.

46. Logacheva MD, Penin AA, Valiejo-Roman CM, Antonov AS. Structure and Evolution of Junctions between Inverted Repeat and Small Single Copy Regions of Chloroplast Genome in Non-Core Caryophyllales. Mol. Biol. 2009;43:757-765.

47. Simmonds SE, Smith J F, Davidson C, Davidson C, Buerki S. Phylogenetics and comparative plastome genomics of two of the largest genera of angiosperms, Piper and Peperomia (Piperaceae). Mol Phylogenet Evol. 2021;163:107229.

48. Wen J, Xie DF, Price M, Ren T, Deng Y Q, Gui L J, et al. Backbone phylogeny and evolution of Apioideae (Apiaceae): New insights from phylogenomic analyses of plastome data. Mol Phylogenet Evol. 2021;161:107183.

49. Logacheva MD, Samigullin TH, Dhingra A, Penin AA. Comparative chloroplast genomics and phylogenetics of Fagopyrum esculentum ssp. ancestrale - a wild ancestor of cultivated buckwheat. BMC Plant Biol. 2008;8:59.

50. Logacheva MD, Schelkunov MI, Fesenko AN, Kasianov AS, Penin AA. Mitochondrial genome of Fagopyrum esculentum and the genetic diversity of extranuclear genomes in buckwheat. Plants. 2020;9:618.

51. Sanchez A, Schuster TM, Kron KA. A large-scale phylogeny of Polygonaceae based on molecular data. Int J Plant Sci. 2009;170:1044-1055. 
52. Sanchez A, Schuster TM, Burke JM, Kron KA. Taxonomy of Polygonoideae (Polygonaceae): a new tribal classification. Taxon. 2011;60:151-160.

53. Kawasaki M, Ohnishi O. Two distinct groups of natural populations of Fagopyrum urophyllum (Bur. et Franch.) Gross revealed by the nucleotide sequence of a noncoding region in chloroplast DNA. Genes Genet Syst. 2006;81:323-332.

54. Tsuji K, Yasui Y, Ohnishi O. Search for Fagopyrum species in eastern China. Fagopyrum. 1999;16:16.

55. Chen QF. A study of resources of Fagopyrum (Polygonaceae) native to China. Bot J Linn Soc. 1999;130:53-64.

56. Cheng C, Fan Y, Tang Y, Zhang KX, Joshi DC, Jha R, et al. Fagopyrum esculentum ssp. ancestrale-a hybrid species between diploid F. cymosum and F. esculentum. Front Plant Sci. 2020;11:1073.

57. Ohsako T, Ohnishi O. Nucleotide sequence variation of the chloroplast trnK/matK region in two wild Fagopyrum (Polygonaceae) species, F. leptopodum and F. statice. Genes Genet Syst. 2001;76:39-46.

58. Ma KH, Kim NS, Lee GA, Lee SY, Lee JK, Yi JY, et al. Development of SSR markers for studies of diversity in the genus Fagopyrum. Theor Appl Genet. 2009;119:1247-1254.

59. Mizuno N, Yasui Y. Gene flow signature in the S-allele region of cultivated buckwheat. BMC Plant Biol. 2019;19:125.

60. Doyle J, Doyle JL. Genomic plant DNA preparation from fresh tissue-CTAB method. Phytochem. Bull. 1987;19:11.

61. Jin JJ, Yu WB, Yang JB, Song Y, Depamphilis C, Yi TS, et al. GetOrganelle: a fast and versatile toolkit for accurate de novo assembly of organelle genomes. Genome Biol. 2020;21:241.

62. Kearse M, Moir R, Wilson A, Stones-Havas S, Cheung M, Sturrock S, et al. Geneious Basic: an integrated and extendable desktop software platform for the organization and analysis of sequence data. Bioinformatics. 2012;28:1647-1649.

63. Qu XJ, Moore MJ, Li DZ, Yi TS. PGA: a software package for rapid, accurate, and flexible batch annotation of plastomes. Plant Methods. 2019;15:50.

64. Tillich M, Lehwark P, Pellizzer T, Ulbricht-Jones ES, Fischer A, Bock R. GeSeq - versatile and accurate annotation of organelle genomes. Nucleic Acids Research. 2017;45:W6-W11.

65. Lohse M, Drechsel O, Kahlau S, Bock, R. OrganellarGenomeDRAW-a suite of tools for generating physical maps of plastid and mitochondrial genomes and visualizing expression data sets. Nucleic Acids Res. 2013;41:575-581.

66. Kurtz S, Choudhuri JV, Ohlebusch E, Schleiermacher C, Stoye J, Giegerich R. REPuter: the manifold applications of repeat analysis on a genomic scale. Nucleic Acids Res. 2001;29:4633-4642.

67. Katoh K, Standley DM. MAFFT multiple sequence alignment software version 7: improvements in performance and usability. Mol Biol Evol. 2013;30: 772-780.

68. Frazer KA, Pachter L, Poliakov A, Rubin EM, Dubchak I. VISTA: computational tools for comparative genomics. Nucleic Acids Res. 2004;32:273-279. 
69. Librado P, Rozas J. DnaSP v5: a software for comprehensive analysis of DNA polymorphism data. Bioinformatics. 2009;25:1451-1452.

70. Thiel T, Michalek W, Varshney R, Graner A. Exploiting EST databases for the development and characterization of genederived SSR- markers in barley (Hordeum vulgare L.). Theor Appl Genet. 2003;106:411-422.

71. Benson G. Tandem repeats finder: a program to analyze DNA sequences. Nucleic Acids Res. 1999;27:573-580.

72. Stamakis A. RAxML version 8: A tool for phylogenetic analysis and post-analysis of large phylogenies. Bioinformatics. 2014;30:1312-1313.

73. Miller MA, Pfeiffer W, Schwartz T. Creating the CIPRES science gateway forinference of large phylogenetic trees. In: Gateway Computing Environments Workshop. 2010. Available at: http://www.phylo.org/index.php/.

74. Swofford DL. PAUP*. Phylogenetic analysis using parsimony (* and other methods). Version 4. Sinauer Associates, Sunderland, MA. 2003.

75. Ronquist F, Huelsenbeck JP. MrBayes 3: Bayesian phylogenetic inference under mixed models. Bioinformatics. 2003;19:1572-1574.

\section{Figures}




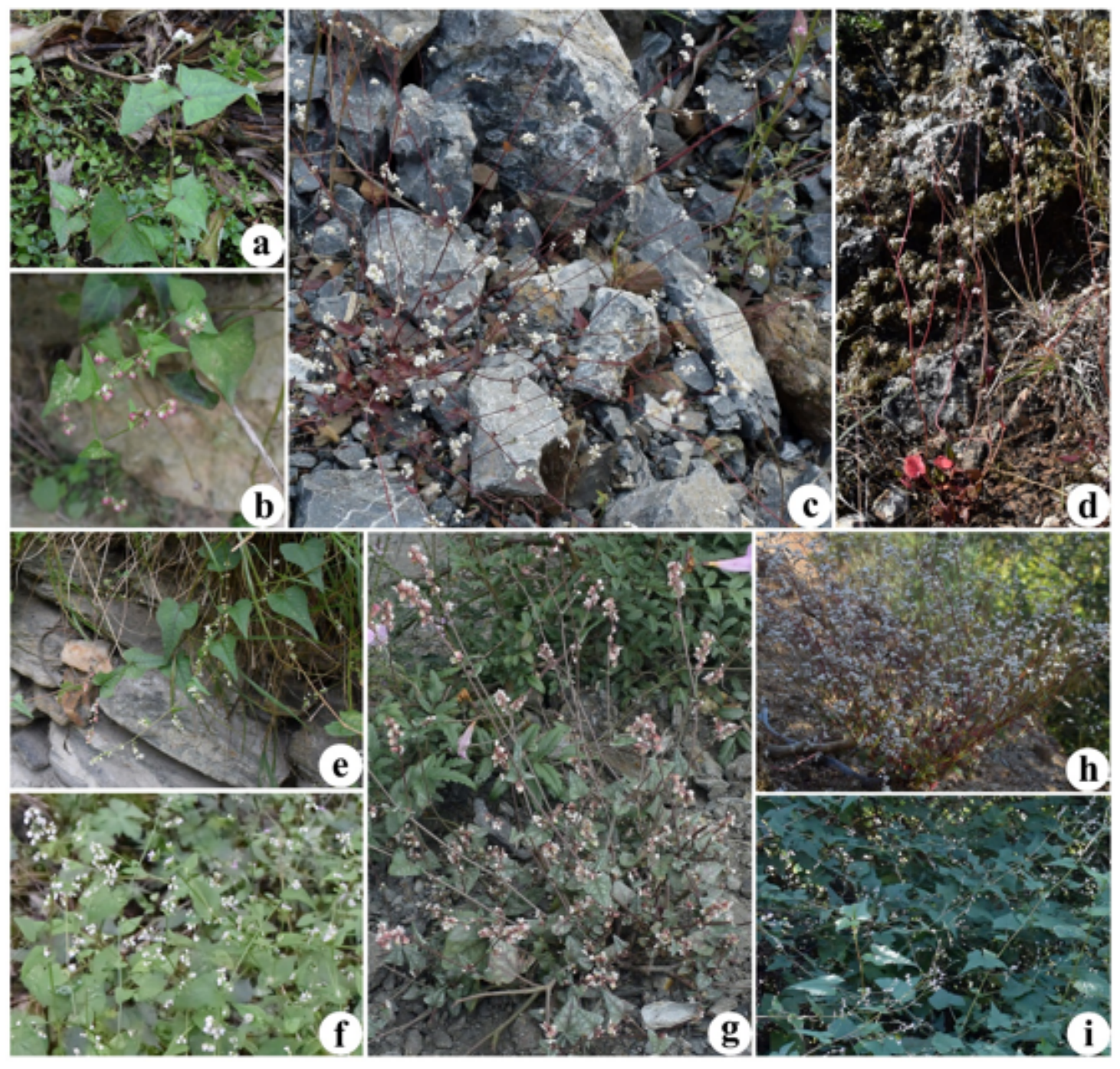

\section{Figure 1}

Field photographs of Fagopyrum taxa show divergent morphological features. a F. cymosum (Photo was taken from Jiguanshan, Chongzhou, Sichuan, China); b F. gracilipes var. odontopterum (Photo was taken from Yangchang, Dafang, Guizhou, China); c F. leptopodum var. grossii (Photo was taken from Jinsha, Leibo, Sichuan, China); d F. statice (Photo was taken from Yumeidu, Shilin, Yunnan, China); e F. caudatum (Photo was taken from Tonghua, Wenchuan, Sichuan, China); f F. gracilipes (Photo was taken from Waziping, Dujiangyan, Sichuan, China); g F. qiangcai (Photo was taken from Bashinao, Lixian, Sichuan, China); h F. leptopodum (Photo was taken from Ala, Panzhihua, Sichuan, China); i F. urophyllum (Photo was taken from Jizushan, Binchuan, Yunnan, China).

\section{Figure 2}

The plastome map of Fagopyrum. Genes shown outside the circle are transcribed clockwise, whereas genes shown inside the circle are transcribed counterclockwise. The color of genes differentiates the protein-coding genes based on their respective functions. The AT and GC contents of the genome are 
plotted on the inner circle as light and dark grey, respectively. The inverted repeats are denoted as IRa and $\mathrm{IRb}$; the large single-copy is denoted as LSC, and the small single-copy regions are denoted as SSC.

\section{Figure 3}

The relevance between the expansion/contraction of LSC-IRs and the plastome-based phylogeny. a Left side is the phylogenetic framework reconstructed using 69 complete plastome sequences. Right side is the length stacked bar chart of the four constituent regions (LSC, IRb, SSC, and IRa) of plastomes. The blue bars represent LSC regions, green bars represent IRs

(IRb and IRa), and brown bars represent SSC regions. $\mathbf{b}$ Right side is the length stacked bar chart of the gene and intergenic regions of plastomes. The black bars represent intergenic regions, and gray bars represent gene regions.

\section{Figure 4}

Phylogenetic relationships of the Polygonaceae, inferred by maximum likelihood (ML) based on complete plastome sequences. Accessions of the species used in the phylogenetic tree are provided in Table S1, S2. Values above branches are bootstrap support (BS). Illustrations represent the junctions of the inverted repeat (IR) and the single-copy (SC) regions. The color blocks in the phylogenetic tree correspond to different types of IR/SC boundaries.

\section{Figure 5}

Phylogenetic informativeness (Pi), insertions/deletions (indels) and polymorphic sites (SNPs) among the plastomes of Fagopyrum. a Sequence diversity of gene regions. b Sequence diversity of intergenic regions. The $\mathrm{X}$-axis shows the regions in plastomes, while the $\mathrm{Y}$-axis represents the Pi, indels and SNPs at each region. The LSC, IR, and SSC on the X-axis indicate large single-copy, inverted repeat, and small single-copy regions, respectively. The gray shaded regions represent the inverted repeat (IR) regions.

\section{Figure 6}

Phylogenetic relationships within the Fagopyrum based on the complete plastome sequences. The size of achenes is on the same scale. Values above branches are posterior probabilities (PP), and values 
below branches are bootstrap support (BS) percentages, inferred from maximum-likelihood analyses (left) and maximum parsimony (right).

\section{Figure 7}

Comparison of repetitive sequences among Fagopyrum species. a Represent short dispersed repeats (SDRs) that present in specific range of size i.e., 30-39 represent numbers of repeats within the size range of 30 and 39 bp. b Describe number of different types of SDRs. $\mathbf{c}$ Represent repeats that present different types of simple sequence repeats (SSRs). $\mathbf{d}$ Represent numbers of different types repeats in 12 Fagopyrum species.

$50 \%$.

\section{Figure 8}

Plastome sequence similarity of Fagopyrum. a The blue squares represent plastome sequence similarity of species in same subclade or between different subclade but in same group. The pink squares represent plastome sequence similarity of species in same group or between different group. $\mathbf{b}$ The blue squares represent plastome gene regions similarity of species in same subclade or between different subclade but in same group. The pink squares represent plastome gene regions similarity of species in same group or between different group.

\section{Supplementary Files}

This is a list of supplementary files associated with this preprint. Click to download.

- Additionalfile1Supplementaryfigureandtable.docx

- Additionalfile2PlastomeDNASequencesofFagopyrum.zip 